\title{
Wundauflagen und Wundspüllösungen
}

\author{
Neue Kennzeichnungs- und Risikobewertungspflicht für PHMB
}

Seit Mitte der 1990er Jahre ist Polyhexanid (PHMB) als Grundstoff für die Zubereitung von Wundspüllösungen zugelassen und wird aufgrund seiner breiten antimikrobiellen Wirkung, so Großkopf et al.: „zur Behandlung von infizierten Wunden oder als Wundprophylaxe genutzt.“

Allerdings wurde PHMB 2011 von der Europäischen Chemikalienagentur (ECHA) als karzinogener Stoff der Kategorie 2 eingestuft (und fällt daher unter den Begriff der CMR-Substanzen, den Sammelbegriff für karzinogen, mutagen oder reproduktionstoxisch) und seit Beginn 2015 gilt für chemische Stoffe und Gemische, die PHMB ab einer Konzentration von 1 Prozent enthalten, eine Kennzeichnungsverpflichtung. Für Medizinprodukte, die invasiv oder mit Körperberührung zum Einsatz kommen gilt zwar eine Ausnahmeregelung, die jedoch mit einer Verpflichtung des Herstellers zum Nachweis der Eignung bzw einer Risikobewertung verknüpft ist.

In Kosmetika ist PHMB, das hier als Konservierungsmittel eingesetzt wird, seit Anfang 2015 im EU-Raum verboten, wird aber bezüglich eventuell ungefährlicher Konzentrationen noch untersucht. Hinsichtlich seiner antimikrobiellen Wirkung weist die Substanz eine große Bandbreite gegen grampositive und gramnegative Bakterien einschließlich multiresistenter Erreger, Pilze und Hefen auf und ist daher als Substanz in Wundauflagen und Wundspüllösungen enthalten. Dabei werden Konzentrationen von 0,3 Prozent nicht überschritten. Die Einstufung als karzinogener Stoff der Kategorie 2, die nun die Kennzeichnungspflicht ab einer Konzentration von einem Prozent zur Folge hatte, stützt sich auf den Verdacht, dass PHMB beim Einatmen giftig ist. „Die Aufnahme von PHMB in die CLP (Classification, labelling and packaging of substances and mixtures) - Verordnung bedeutet nicht automatisch eine Stoffbeschränkung oder ein Verbot", stellt Gerlach fest, sie soll primär den Anwender vor gefährlichen Stoffen und Gemischen schützen: „Die rechtliche Konsequenz ist die verpflichtende Kennzeichnung ab einer Konzentration von 1 Prozent als ,Carc. 2“'; bei Gemischen, die PHMB ab einer Konzentration von 0,1 Prozent enthalten, muss ein Vermerk im Sicherheitsdatenblatt erfolgen.

\section{Der Hersteller belegt das Sicherheitsrisiko}

Die Ausnahme von der Kennzeichnungspflicht für Medizinprodukte, die invasiv oder mit Körperkontakt angewendet werden, bedeutet jedoch, so Großkopf et al., dass sich die Bewertung nach den entsprechenden nationalen oder EU-Vorschriften des Medizinproduktegesetzes richtet: „Der deutsche Gesetzgeber hat ... hervorgehoben, dass die Eignung des betreffenden Medizinproduktes zu dessen vorgesehenen Verwendungszweck grundsätzlich vom Hersteller anhand von klinischen Daten zu legen ist." Dabei ist eine entsprechende fundierte Nutzen-RisikoAbwägung aufzustellen. Damit soll der Sicherheitsaspekt zwingend und, mit den entsprechenden verfügbaren wissenschaftlichen Erkenntnissen belegt, dokumentiert werden. „Im Rahmen der Risikobewertung ist der Hersteller von Medizinprodukten auch verpflichtet, bei als gefährlich eingestuften Stoffen, insbesondere CMRSubstanzen stets zu prüfen, ob für deren Einsatzgebiet ein gefahrloser alternativer Stoff zur Verfügung steht", berichtet Gerlach.

\section{LITERATUR}

Großkopf V. et al.: Polihexanid - Rechtsaspekte einer Risioeinschätzung, in: Rechtsdepesche für das Gesundheitswesen, Mai/Juni 2015

Gerlach S.: Kennzeichnung von Medizinprodukten, in Das Medizinprodukt 2/15

\footnotetext{
IMPRESSUM

Herausgeber und Verleger: Springer-Verlag GmbH, Professional Media, Prinz-Eugen-Str. 8-10, Postfach 11, 1040 Wien, Austria, Tel.: 01/330 24 15, Fax: 01/33024 26 Internet: www.springer.com, www.SpringerMedizin.at Geschäftsführung: Dr. Alois Sillaber, Petrus J.W. Hendriks, Joachim Krieger Leitung: Dr. Alois Sillaber Leitung Journale und Redaktionen Medizin: Gabriele Hollinek Redaktion: Verantwortlicher Redakteur: Verena Kienast Mitarbeiter dieser Ausgabe: Dipl. Tzt. Elise Haidenthaller Redaktionssekretariat: Susanna Hinterberger Produktion: Dorothea Wolinski Leitung Verkauf Medizin: Robert Seiwald Anzeigen: Gabriele Popernitsch; Es gilt die Anzeigenpreisliste 2015. Erscheinungsweise: 10× jährlich Abonnement: Michaela Bolli; Bezugspreis pro Jahr: für Institutionen EUR 109,- zuzüglich MwSt. und Versandkosten Bezugsbedingungen: Das Abonnement für Einzelbezieher gilt mit Bezug des ersten Heftes jeweils für ein Jahr mit der in der Preisliste für einen vollen Jahrgang angegebenen Anzahl von Ausgaben. Abbestellungen innerhalb dieser Laufzeit können nicht entgegengenommen werden. Das Abonnement der Zeitschrift verlängert sich automatisch um ein weiteres Jahr, wenn nicht bis 2 Monate vor Ablauf des Abonnements beim Verlag eine schriftliche Kündigung eingegangen ist. Verlagsort: Wien Herstellungsort: Linz Erscheinungsort: Wien Verlagspostamt: 1040 Wien P. b. b. ISSN Print: 0949-7323, ISSN Electronic: 1613-7574, Band 20, Heft 6-7/2015 Layout: KM-Sagso KM-Satz Design: Wojtek Grzymala Druck: Friedrich Vereinigte Druckereien- und Verlags GmbH \& Co KG, Linz, Austria Wissenschaftlicher Beirat: FH-Prof. Dr. Holger Penz, Feldkirchen;

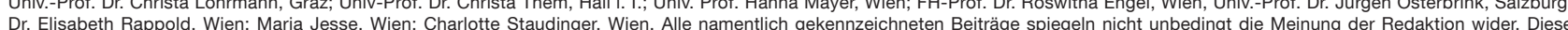
Dr. Elisabeth Rappold, Wien; Maria Jesse, Wien; Charlotte Staudinger, Wien. Alle namentlich gekennzeichneten Beiträge spiegeln nicht unbedingt die Meinung der Redaktion wider. Diese Beitrage fallen somit in den personlichen Verantwortungsbereich des Verfassers. Die Redaktion übernimmt keine Haftung fur unaufgefordert eingesandte Manuskipte. Mit "Sonderbericht" ode „Advertorial“ gekennzeichnete Seiten sind entgeltliche Einschaltungen nach $\$ 26$ Mediengesetz. Allgemeiner Teil/Rechtliche Hinweise für Autoren Die Autorin/der Autor erklärt, dass ihr/sein Manuskript in dieser Form bislang nicht anderweitig veroffentlicht oder zur Veroffentlichung eingereicht wurde. Die Autorin/der Autor ubertragt michung in der Fachzeitschrift die notwendigen Nutzungsrechte zur Vervielfältigung und Verbreitung an den Verlag, insbesondere das Recht der Nutzung zu gewerblichen Zwecken der Veröffentlichung in der Fachzeitschrift die notwendigen Nutzungsrechte zur Vervielfältigung und Verbreitung an den Verlag, insbesondere das Recht der Nutzung zu gewerblichen Zwecken durch Druck, Nachdruck, Verbreitung in elektronischer Form oder andere Verfahren und Medien durch Springer Science + Business Media. Beiträge, die in PROCARE erscheinen, können auch in der Springer-Zeitschrift Heilberufe veröffentlicht werden. Die Autorin/der Autor holt, falls notwendig, die Nutzungsrechte an Texten und Bildern Dritter vor Ubergabe des fertigen ManuSkripts ein, eventuelle Ansprüche Dritter sind somit geklärt. Hinweise zur Verwertung: Die Zeitschrift sowie alle in ihr enthaltenen einzelnen Beiträge und Abbildungen sind urheberrechtlich
geschützt. Jede Verwertung, auch auszugsweise, die nicht ausdrücklich vom Urheberrechtsgesetz zugelassen ist, bedarf der vorherigen schriftlichen Zustimmung des Verlages. Das gilt insbegeschützt. Jede Verwertung, auch auszugsweise, die nicht ausdrücklich vom Urheberrechtsgesetz zugelassen ist, bedarf der vorherigen schriftlichen Zustimmung des Verlages. Das gilt insbesondere für Vervielfältigungen, Bearbeitungen, Ubersetzungen, Mikroverfilmungen und die Verarbeitung in elektronischen Systemen. Produkthaftung: Die Wiedergabe von Gebrauchsnamen,
Handelsnamen, Warenbezeichnungen usw. in dieser Zeitschrift berechtigt auch ohne besondere Kennzeichnung nicht zu der Annahme, dass solche Namen im Sinne der Warenzeichen- und Markenschutz-Gesetzgebung als frei zu betrachten wären und daher von jedermann benutzt werden dürften. Angaben über Dosierungsanweisungen und Applikationsformen sind anhand anMarkenschutz-Gesetzgebung als frei zu betrachten wären und daher von jedermann benutzt werden dürften. Angaben über Dosierungsanweisungen und Applikationsformen sind anhand anWien. Springer ist Teil von Springer Science + Business Media. Coverbild: @ monkeybusinessimages / Getty Images / thinkstock
} 